\title{
Prevention of HBV exposure and infection among gay men and other men who have sex with men (MSM)
}

\section{Abstract}

It is a well-known fact that around 20 to $25 \%$ of new hepatitis B cases occur among men who have sexual intercourse with other men (MSM), whether they identify as gay or not. This excessive burden is related to several situations that require to be urgently addressed. Among the most important:

i. The high viral load of HBV in semen among chronic carriers;

ii. The very poor efforts undertaken by health authorities, institutions and organizations to raise awareness about the existence and severity of the problem among MSM populations and groups (gay, bisexual, or self-identified as heterosexuals with sexual intercourse with other men);

iii. The possibility of frequent exposure can be associated to greater odds of acquiring HBV and of developing chronic infection and

iv. The insufficient commitment and resources allocated to facilitate access to HBV vaccination by MSM.

Background: Hepatitis B virus is shed in the semen of chronic surface antigen (HBsAg) carriers as DNA viral particles. Since HBV is a very infectious agent, exposure to this agent may result in an efficient transmission and further spreading of the infection. Although most people who have acquired the infection will clear the virus without any medical intervention, it is estimated that between 5 to $10 \%$ will progress to chronic infection. Chronic infection is associated to health conditions that vary in severity from long-term hepatitis to fulminant hepatic failure and/or hepatic cancer. The disproportionate toll of HBV infection MSM echoes the also disproportionate toll of the HIV epidemic among this population that encompasses diverse groups and subpopulations. Given that co-infection with HBV and HIV may result in complex situations with negative public health and clinical outcomes. Thus, efforts to promote primary prevention of infection with both agents, reduction of incidence of coinfections, and active detection of the presence of HIV and HBV are of paramount importance. ${ }^{1}$

While HIV is a rather well-known topic within the MSM communities and groups, there seems to be a diminished awareness about HBV (and other viral hepatitis) infections. Knowledge about HBV infection and strategies and practices to prevent or keep it under clinical control by gay men and other MSM doesn't seem to have been deeply investigated. At least not in developing countries. In areas where hepatitis A is endemic, many people may assume that other hepatitis can be cleared by the system spontaneously as it does with HAV. Many MSM may even ignore that HAV is transmitted by contact with contaminated fecal residues and give little or no importance to sexual practices that involve exposure to feces. In conversations with gay men in Brazil, they describe the practice of "writing a check", which is the withdrawal of a feces-covered penis from the anus of the receptive partner. Since this practice does not necessarily entails using a condom it may happen that the receptive partner passes $\mathrm{HAV}$ in his fecal particles while the penetrator transmits HBV in his sperm. In courses and seminars about health care for gay men, the topic of viral hepatitis arises as a big surprise for many members of the audiences. Yet, nothing can be said until the level of awareness and knowledge is more systematically quantified. ${ }^{2}$
Volume 4 Issue I - 2018

Rafael Mazin

Independent Consultant, USA

Correspondence: Rafael Mazin, MD; MPH: Independent Consultant; Former Senior Advisor on HIV, STI and viral hepatitis, Pan American Health Organization/Regional Office of the World Health Organization, I 3007 Davenport Drive Brandywine, Maryland, USA, 206।3, Tel I 202 2553। I8, Email mazinraf@gmail.com

Received: November 26, 2017 | Published: February 09, 2018

\section{Incomplete responses}

International organizations, agencies and institutions have made a strong call for early diagnosis of HIV and the prompt -or even immediate-initiation of antiretroviral treatment. It doesn't happen the same with viral hepatitis. Hepatitis B can't be cured yet. There is no effective treatment to clear the infection among chronic carriers. Yet, being aware of the condition of chronic carrier allows to make changes in one's life and to prevent the dissemination of the infection. 
Some of the strategies in the so called "combined prevention against HIV infection" are equally effective to halt HBV (Condom use and other forms of safe sex)

Condom use and other forms of safe sex. Recently an approach based on the use of two antiretroviral drugs (emtricitabine and tenofovir disoproxil fumarate) is being used as a strategy to prevent transmission. This strategy is known as pre-exposure prophylaxis or PrEP and has proven to be highly effective (reduction of $92 \%$ in risk of transmission). Still, its efficacy is centered in preventing HIV transmission and no known effect on other STIs. In developed countries it is likely that providers inform the users about this fact. This does not seem to be the case in developing countries where the basics about PrEP are poorly communicated and taught, even among providers. $^{3}$

It must be mentioned that the combinations of the two PrEP drugs influence HBV metabolism and it has been said to cause hepatitis B flares among users whether at the initiation of treatment or during periodical interruptions. However, during the conduction of the iPrEx no flares were observed among participants with chronic HBV infection. ${ }^{4}$

It is very disappointing the realization that closes to zero attention is given to prevention strategies and methods against $\mathrm{HBV}$ in recent literature and academic meetings. In a review of the proceedings of international gathering of experts in hepatitis and other liver ailments held during 2017 most of discussions was about improvement of treatments. The few discussions about prevention gravitated around prevention of mother to child transmission. While both fields are of enormous importance they completely eclipsed the urgent need to focus prevention efforts on most impacted populations and groups, among which MSM.

It is also regrettable that some leaders in the public health effort to deal with the viral hepatitis epidemics show great disdain for actions directed to raise awareness, increase knowledge and trigger behavioral changes for reducing the risk of exposure to the causing viruses, augment health-seeking behaviors and contribute to contain the spreading of the epidemic. Unfortunately, there are still many "experts" who want to address health issues in communities only with bio-medical responses.

The best avenue to address this deficiency and thus clear institutional hurdles would be to increase the knowledge of community members and expand their capacity to educate their peers about the need to exclude HBV from a healthy a pleasant sexual life and wellbeing.

Vaccination against HBV (and formulations combined to prevent $\mathrm{HAV}$ as well) is widely available. They are very safe, provide effective immune protection, and in the long term will contribute to eradication and even elimination of hepatitis B.

In many countries in the world, vaccination against hepatitis B is given to infants. In the region of the Americas it has been in place for at least 15years. This means that in few years there will be large cohorts of individuals who will be completely immune to transmission of HBV.

In the meanwhile, there are many individuals 20years and older who don't have immune protection and are at risk of exposure and acquiring HBV through sexual intercourse. In a study in Amsterdam by Robin van Houdt, the median age for primary HBV infection among gay men was 31 years. This means that there is still an enormous gap between the generations being vaccinated against HBV and those getting infected.

Regional and national programs for the prevention and control of viral hepatitis make a strong case for universal vaccination of people in the so-called "key populations" (term loaned from the HIV programs that refers to segments of the population with prevalence rates of infection well above the "general population"). It is, however, very disappointing to realize that the specific actions at national and local level for expanding coverage of HBV vaccine for MSM are not in place. A common argument is that the scarce stock of vaccines is limited for the use of medical students and providers, in addition to new-borns and infants. The international health agencies could have collaborated to contour this impasse.

\section{Conclusion}

The disproportionate toll of HBV infection among MSM must be addressed urgently, lest the unwanted consequences and impact of chronic infections add to the entire heavy burden on the health and wellbeing of this population. Strong advocacy efforts should be carried out to bring attention to this health issue and awareness must be raised among the affected population. The "packages" of prevention must be offered as comprehensive approaches rather than exclusive for HIV. Educational efforts must be doubled to ensure MSM are empowered to face the challenge of viral hepatitis along with other STIs and HIV. Furthermore, prevention practices can be taught to heterosexual couples who engage in anal sex and may place the receptive partner (the woman) at increased risk for contracting HBV.

\section{Last, but not least}

Access to $\mathrm{HBV}$ or even better to $\mathrm{HBV} / \mathrm{HAV}$, vaccination must be ensured for the communities as part of comprehensive packages of prevention and care. This access must be effective rather than a simple recommendation on guidelines or in regional, global or national programs. For MSM who does sex work it is important to ascertain their overall health needs, including the presence of $\mathrm{HCV}$ infection. It has been said that "hepatitis is an invisible epidemic". For MSM this argument is merely rhetoric, "lip service", because they are not realizing about it and they still seem to be "invisible" to certain programs and many health care services.

\section{Acknowledgements}

None.

\section{Conflict of interest}

Author declares that there is no conflict of interest.

\section{References}

1. Jenison SA, Lemon SM, Baker LN, et al. Quantitative Analysis of Hepatitis B Virus DNA in Saliva and Semen of Chronically Infected Homosexual Men. J Infect Dis. 1987;156(2):299-307.

2. Pitasi MA, Bingham TA, Sey EK, et al. Hepatitis B Virus (HBV) Infection, Immunity and Susceptibility Among Men Who Have Sex with Men (MSM), Los Angeles County, USA. AIDS Behav. 2014;18(3):248-255. 
3. Solomon MM, Schechter M, Liu AY, et al. (Grant R presenting) The safety of HIV pre-exposure prophylaxis in the presence of hepatitis B infection. 8th International AIDS Society Conference on HIV Pathogenesis, Treatment, and Prevention (IAS 2015), Vancouver, abstract TUAC0201 2015 .
4. van Houdt R, Bruisten SM, Speksnijder AG, et al. Unexpectedly high proportion of drug users and men having sex with men who develop chronic hepatitis B infection. J Hepatol. 2012;57(3):529-533. 